\title{
ABUNDANCIA Y ESTRUCTURA DE LA ICTIOFAUNA EN LA PLATAFORMA DEL LITORAL ROCOSO EN LA ZONA DE PROTECCIÓN ECOLÓGICA -TAUCA, HUACHO
}

\author{
ABUNDANCE AND STRUCTURE OF ICTIOFAUNA AT THE ROCOSO LITTORAL \\ PLATFORM IN THE AREA OF ECOLOGICAL PROTECTION -TAUCA, HUACHO \\ Luis Alberto Cárdenas Saldaña', Ramón León Yovera', \\ William Guzmán Sanchez', José Luis Romero Bozzetta ${ }^{1}$ Alfonzo Diaz Guzman²
}

\begin{abstract}
RESUMEN
El objetivo fue determinar la diversidad y abundancia de la ictiofauna del arrecife rocoso de la Zona de Protección Ecológica de Huacho que es conjunto de especies de peces que existen en una determinada región biogeografía. En la metodologia se uso las Áreas Marinas y Costeras Protegidas que son definidas como áreas geográficas específicas, las cuales han sido designadas para mejorar la conservación de recursos naturales marino-costeros. La zona de estudio está comprendida por la Plataforma del Litoral Rocoso en la Zona del Protección Ecológica-Tauca en Huacho. Que está ubicada en las coordenadas $77^{\circ} 36$ '24" de longitud oeste y $11^{\circ} 06$ '22" de latitud sur al norte de Lima frente al Océano Pacífico, sobre la margen izquierda del río Huaura desde la punta la Viuda hasta Hornillos. Para la identificación de las especies se usó las artes de pesca con nailon desde la orilla y la abundancia mediante preguntas a los pescadores de pinta (de la orilla). En los resultados se encontraron 17 especies siendo la más abundante la Lorna seguido de la chita y luego los borrachos, los Tramboyos y la corvina, y se concluyo que las Lornas y las chitas están con sus hueveras en una talla pequeña, produciéndose lo que se llama la juvenación que no es más que la defensa de estas especies para poder existir.
\end{abstract}

Palabras clave: Ictiofauna; Arrecife rocoso; Diversida; Abundancia.

\begin{abstract}
The objective was to determine the diversity and abundance of the ichthyofauna of the rocky reef of the Ecological Protection Zone of Huacho, which is a set of fish species that exist in a specific biogeographic region. In the methodology, the Marine and Coastal Protected Areas were used, which are defined as specific geographic areas, which have been designated to improve the conservation of marine-coastal natural resources. The study area is comprised by the Rocky Coast Platform in the Ecological Protection Zone-Tauca in Huacho. It is located at coordinates $77^{\circ} 36^{\prime} 24$ "west longitude and $11^{\circ} 06^{\prime} 22^{\prime \prime}$ south latitude north of Lima in front of the Pacific Ocean, on the left bank of the Huaura river from Punta la Viuda to Hornillos. For the identification of the species, fishing gear with nylon from the shore and abundance was used by means of questions to the pint fishermen (from the shore). In the results 17 species were found, the Lorna followed by the cheetah and then the drunkards, the Tramboyos and the croaker, and it was concluded that the Lornas and the cheetahs are with their egg cups in a small size, producing what is it calls the juvenación that is not more than the defense of these species to be able to exist.
\end{abstract}

Keywords: : Ichthyoids fauna; Rocky reef; Diversity; Abundance.

\footnotetext{
${ }_{1}^{1}$ Facultad de Ciencias, Universidad Nacional José Faustino Sánchez Carrión (Lima - Perú). Email: vinvestigacion@unjfsc.edu.pe
} 


\section{INTRODUCCIÓN}

En ecología y en ciencias pesqueras, se llama ictiofauna al conjunto de especies de peces que existen en una determinada región biogeográfica. Así, nos podemos referir, por ejemplo, a la ictiofauna del Tauca que es la Zona de Protección Ecológica de Huacho.

La actividad humana como las construcciones de canales la navegación marítima y fluvial, la pesca excesiva, los desagües, en general las actividades humanas saltaran el entorno y modifican drásticamente la ictiofauna de un lugar concreto.

La definición de arrecife depende de la perspectiva o disciplina en la que se trabaje. Desde el punto de vista ecológico un arrecife es cualquier estructura rocosa sumergida que provee de un substrato duro para el desarrollo de la vida marina. Desde este punto de vista existen varios tipos de arrecifes los coralinos, rocosos y artificiales. (Reyna, 2014)

Varios son los factores que influyen en la distribución de las especies en los arrecifes; destaca la importancia de la disponibilidad de recursos, espacio, refugio y alimento, la topografía del substrato, el grado de exposición de un lugar con respecto a corrientes y oleaje, transparencia del agua, y algunos patrones de comportamiento propios de las especies. (Kleine, 2014)

Concretamente los peces de arrecife son aquellos que tienen su vida íntimamente asociada con el substrato rocoso con fines de alimentación, refugio y/o reproducción. La riqueza de estos hábitats depende no sólo de la productividad primaria y los procesos intrínsecos de la cadena trófica inter mareal, sino también de la productividad que llega a la zona por otras vías tales como las olas y las corrientes procedentes del submareal adyacente y las zonas pelágicas próximas, y en menor medida, de la productividad de los recursos terrestres. (Gil, 2006)

Los peces de arrecife tienen gran importancia tanto desde el punto de vista ecológico, ya que forman parte de una comunidad altamente diversa y compleja, como desde el punto de vista económico por la pesca y el turismo.

La zona litoral se caracteriza porque constantemente está azotada por las olas e influenciada por los cambios de nivel del agua debido a las mareas. Los recursos que habitan esta zona deben adaptarse a este permanente movimiento del mar, por la cual la mayoría de ellos han desarrollado ventosas, como el pejesapo que les permite adherirse a las rocas. (GEF-ONUDI-IMARPEIFOP, 2002)

Hasta el presente en nuestro conocimiento, no hemos encontrado ningún trabajo que haya tratado de estimar las abundancias y la diversidad de las poblaciones de peces del rocoso de la zona de protección ecológica de huacho, algo que resulta imprescindible a la hora de realizar valoraciones del estado en que se encuentra una zona de protección.

\section{MATERIAL Y MÉTODO}

Las Áreas Marinas y Costeras Protegidas son definidas como áreas geográficas específicas, las cuales han sido designadas para mejorar la conservación de recursos naturales marino-costeros. La zona de estudio esta comprendida por la Plataforma del Litoral Rocoso en la Zona del Protección Ecológica-Tauca en Huacho. Que está ubicada en las coordenadas $77^{\circ} 36^{\prime} 24^{\prime \prime}$ de longitud oeste y $11^{\circ} 06^{\prime} 22$ " de latitud sur al norte de Lima frente al Océano Pacífico, sobre la margen izquierda del río Huaura. El área de estudio está conformada desde la punta la Viuda hasta Hornillos. (Figura No1). (INDECI, 2007).

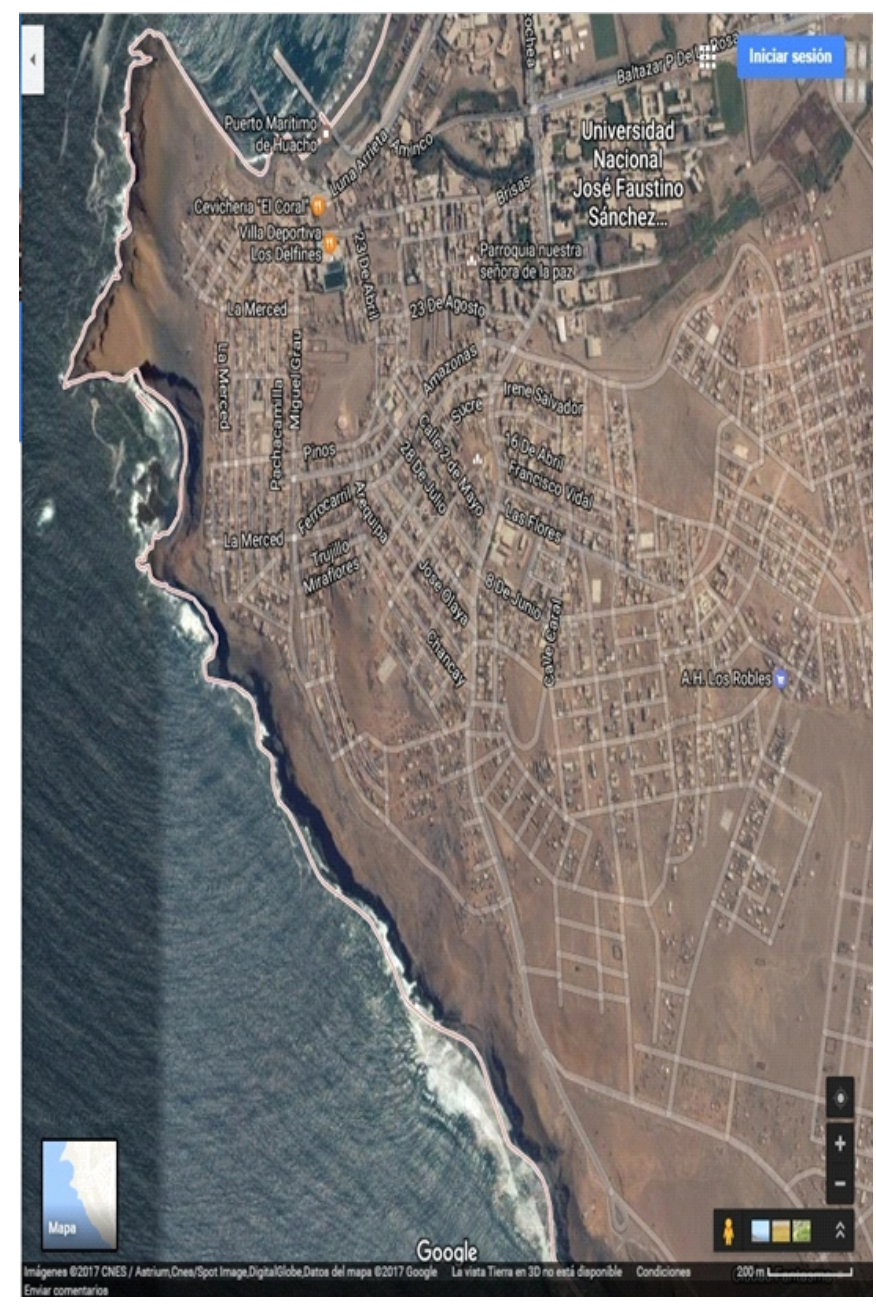

La geomorfología del área destaca por ser de costa abrupta, casi vertical con una zona intermareal estrecha que alcanza entre 5 a $20 \mathrm{~m}$ de profundidad en una distancia de 20 a100 m desde la línea del intermareal.

Para la toma de datos del número de especies de peces en la zona de protección ecológica, se utilizará el método de las artes de pesca y la abundancia se realizó mediante una entrevista a los pescadores artesanales (los pinteros).

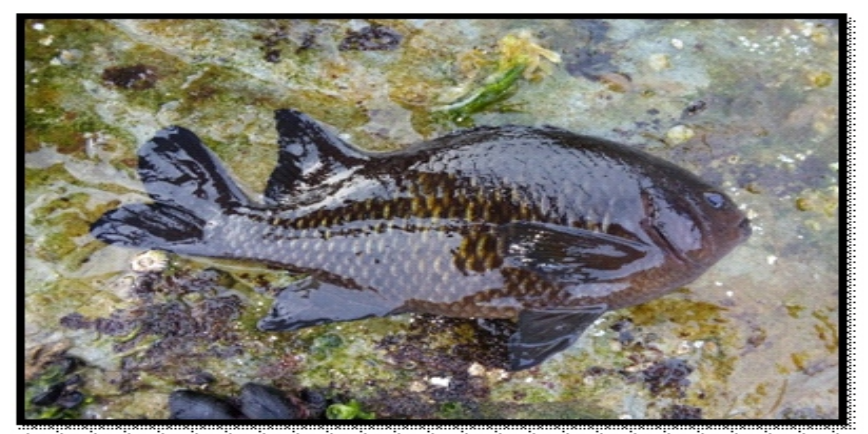

Figura 1. Tetra Viuda Negra - Gymnocorumbus ternetti 


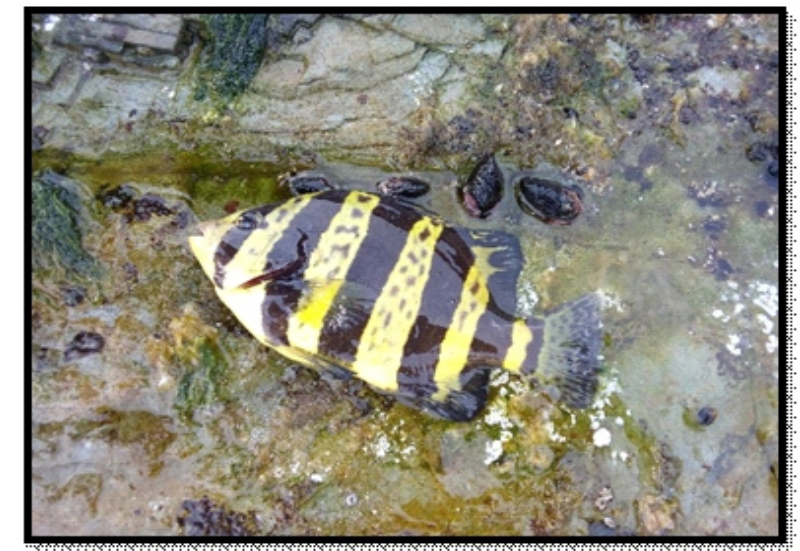

Figura 2. Ciclido cebra

Tilapia buttikoferi

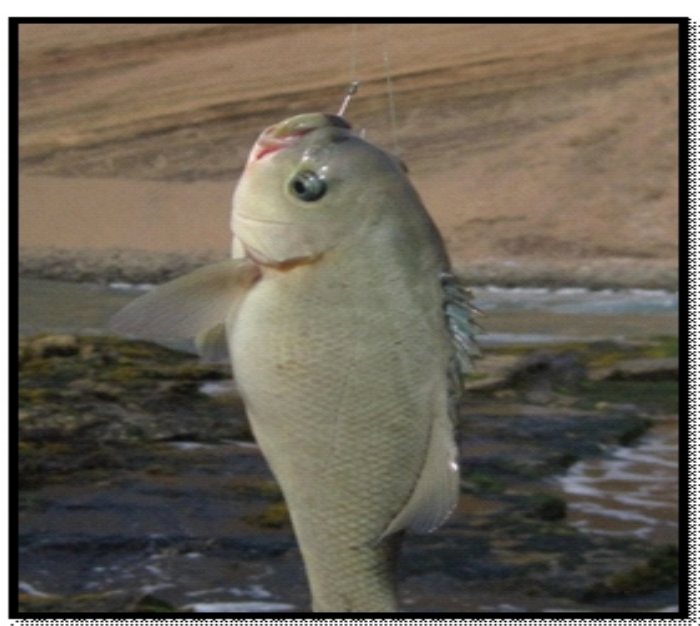

Figura 3. Mero

Epinephelus analogus

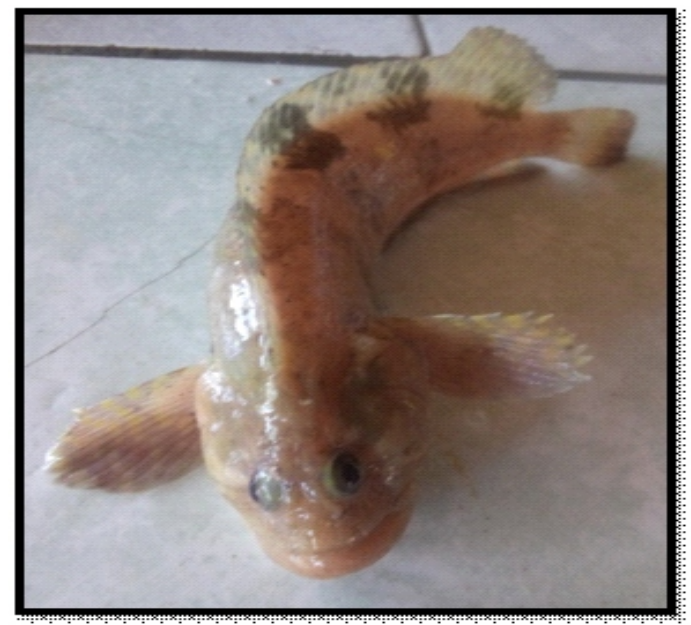

Figura 4: Soñador

Cyprinadon diabolis

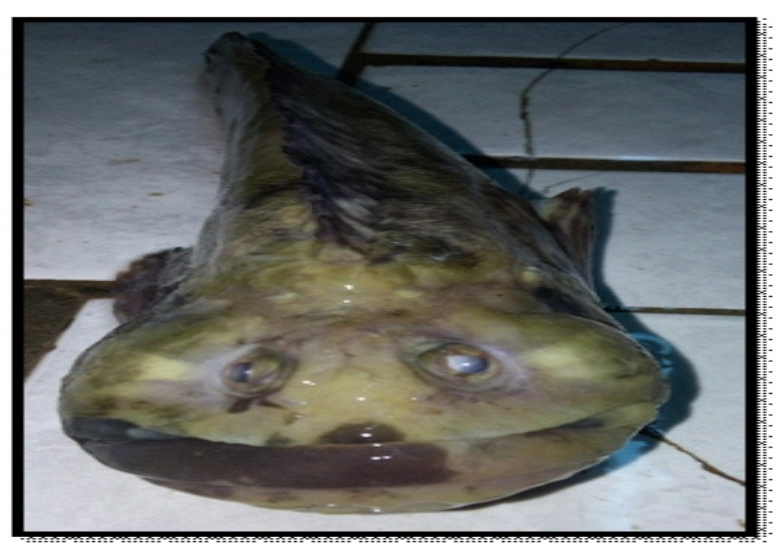

Figura 5. Pejesapo

Sicyases Sangeneus

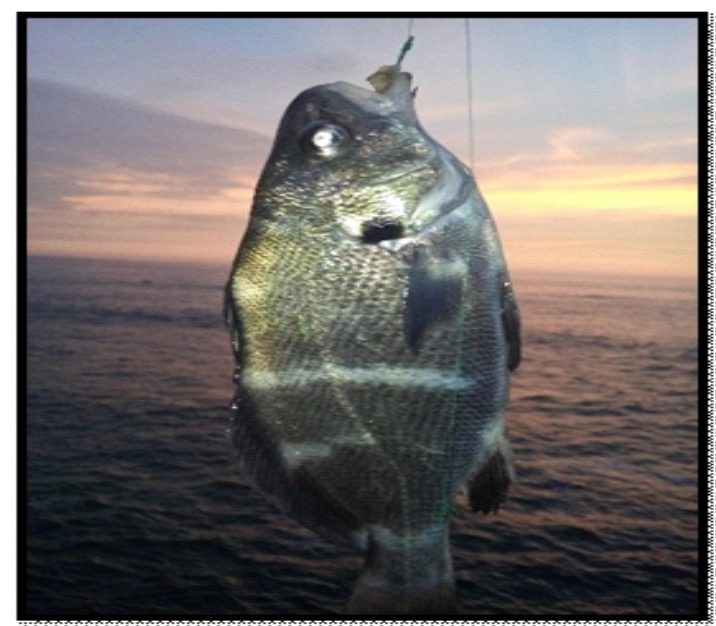

Figura 6. Anillo

Diplodus annularis

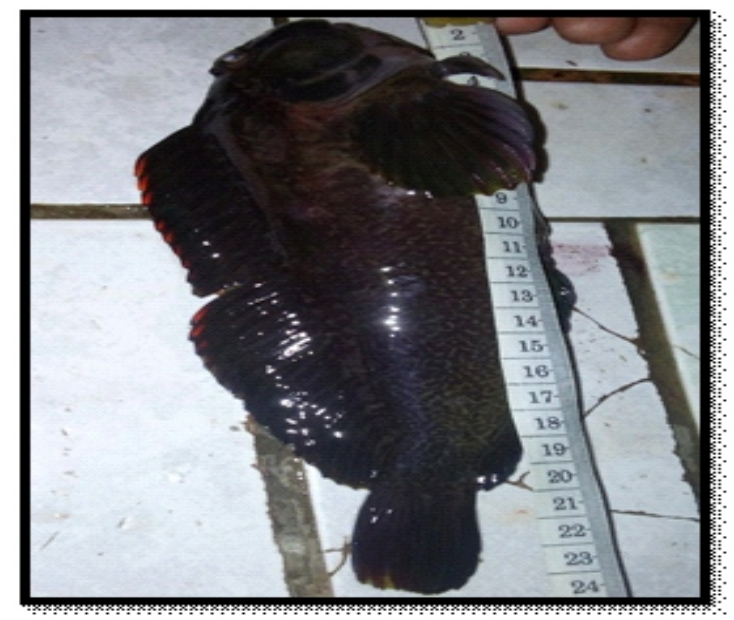

Figura 7:Borracho

Scartichthys gigas 


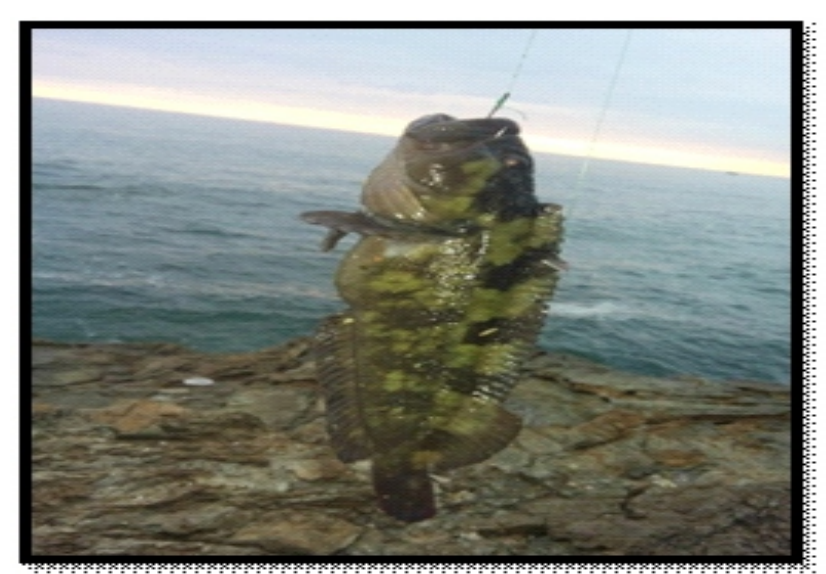

Figura 8: Tranboyo

Labrisomus multiporosus

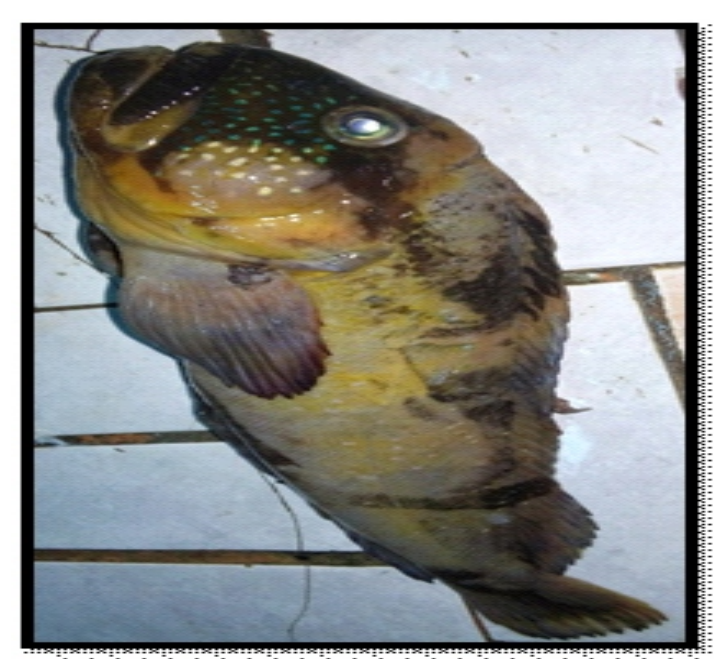

Figura 9: Bocon moteado

Opistognathus maxillasus

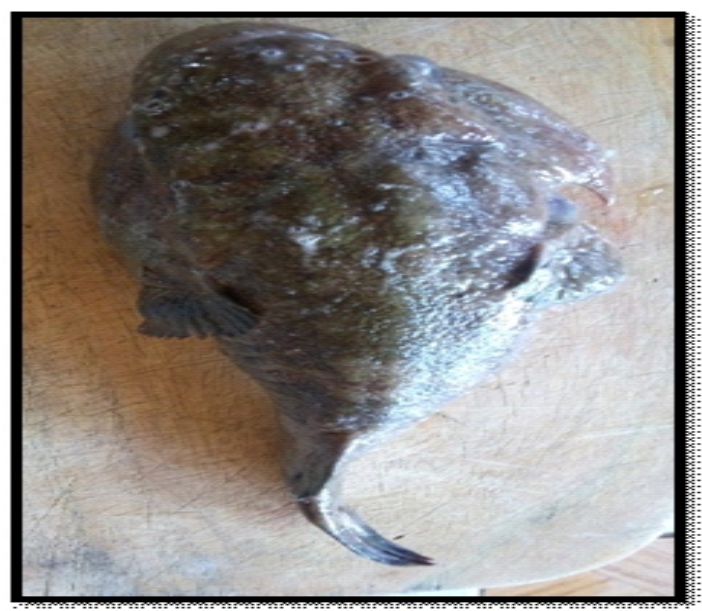

Figura 10: Rape blanco

Lophius piscatorius

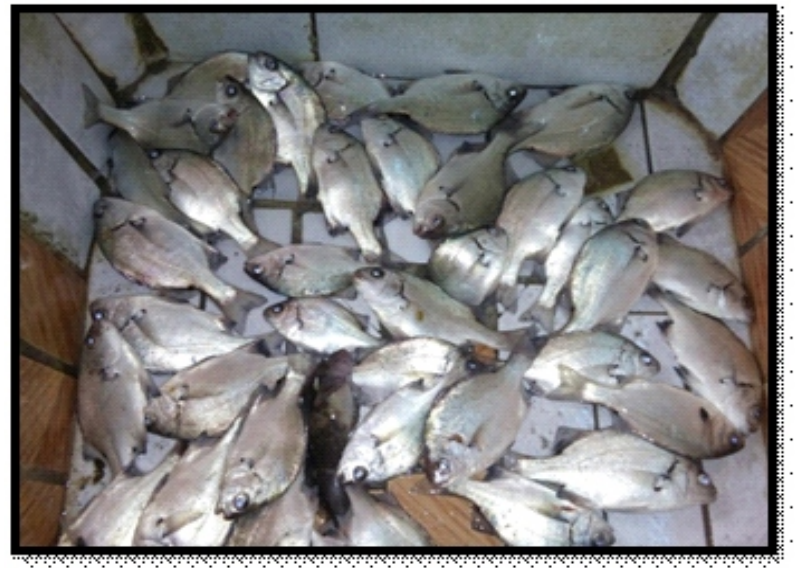

Figura 11: Chita

Anisotremus scapularis

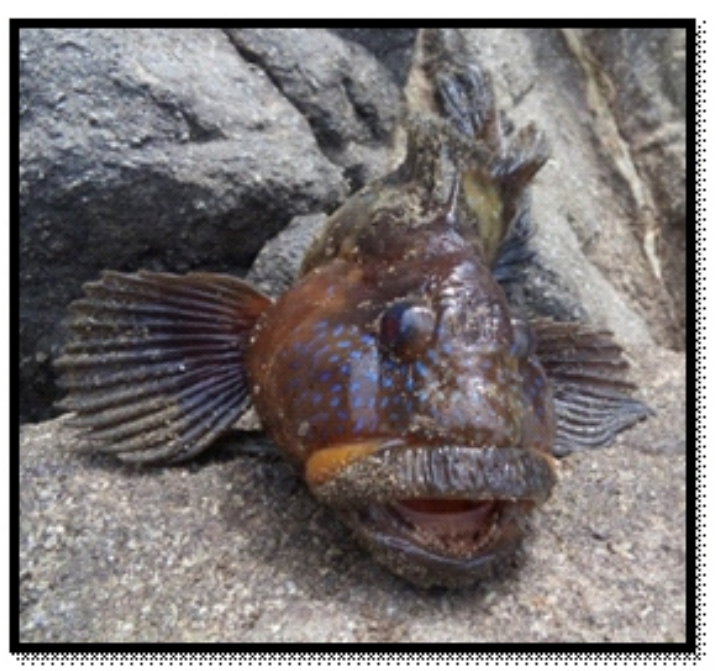

Figura 12: Tramboyo pintado

Labrisomidae labrisomus

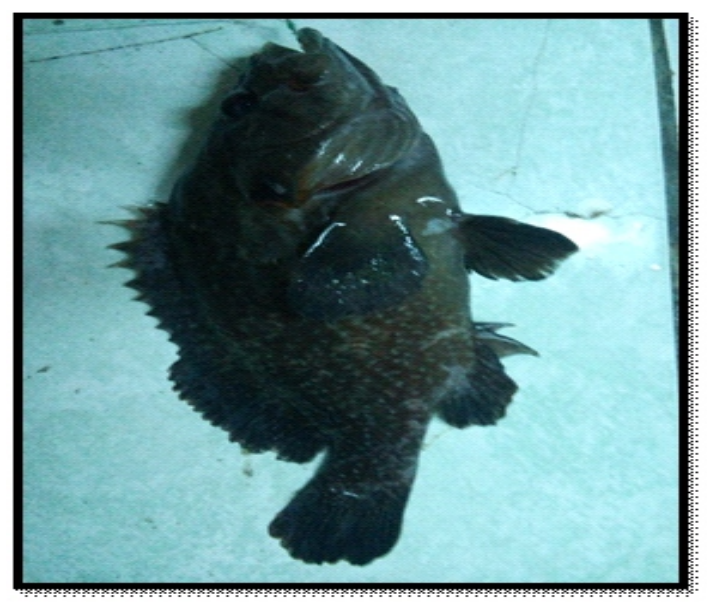

Figura: Cherlo

Acanthistius pictus 


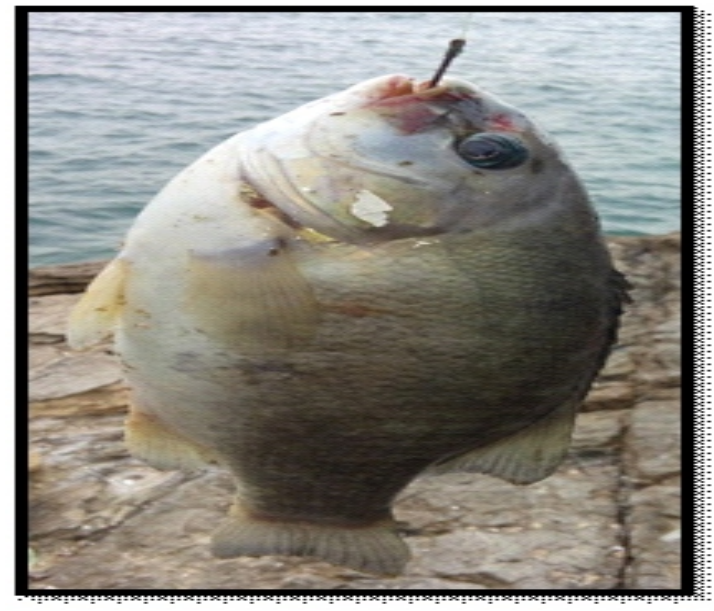

Figura 14: Burro

Plectorhinchus mediterraneus

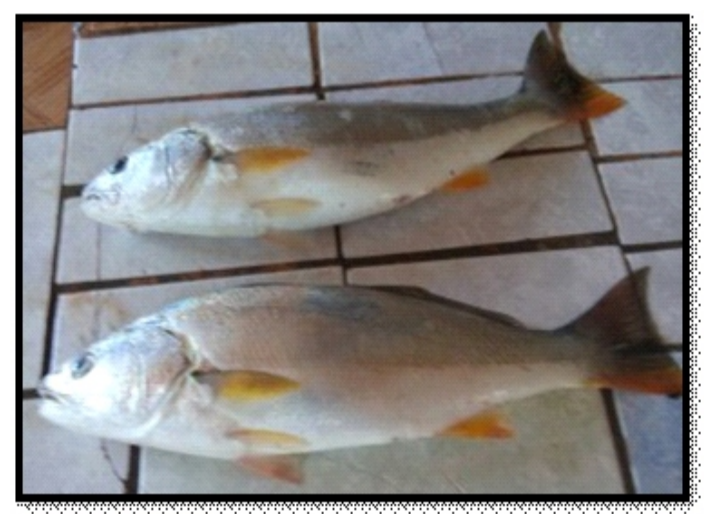

Figura 15: Corvina

Argyrosomus regios

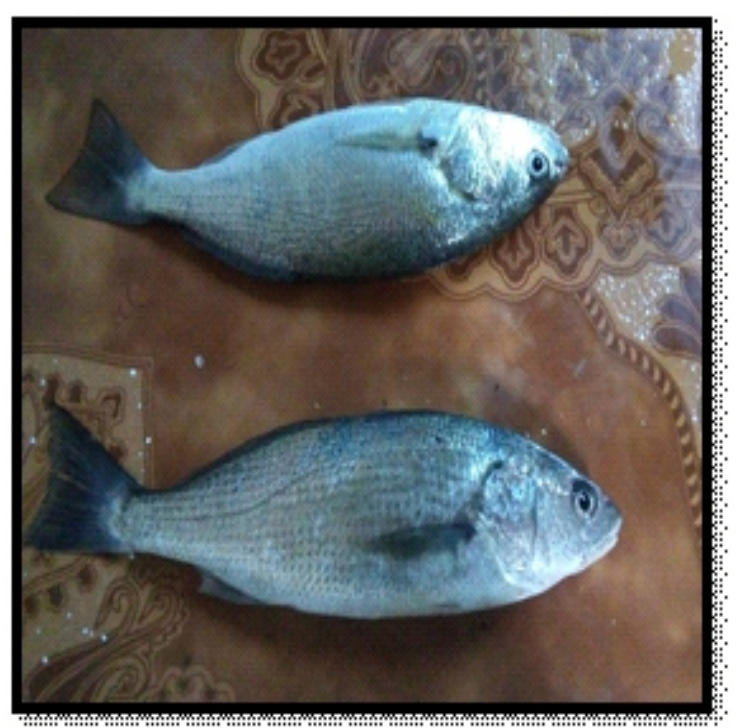

Figura 16: Lorna

Siaena deliciosa

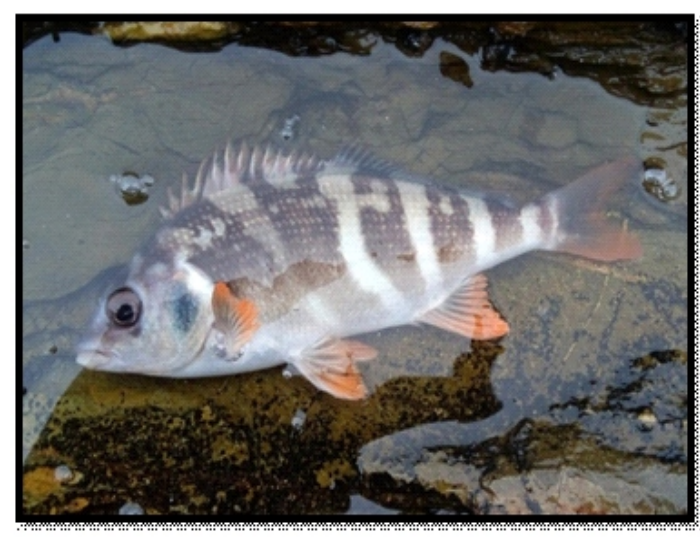

Figura 17: Pintadilla

Cheylodactylus variegatus

\section{DISCUSION}

Generalmente, se consideran peces intermareales aquellos cuya vida se desarrolla en la zona intermareal y para ello poseen una morfología particular, una fisiología propia, y unas adaptaciones etológicas que les permiten sobrevivir en este medio. Las poblaciones combinadas de estas especies, pueden considerarse como la comunidad intermareal, tomando la definición de comunidad como "un ensamblaje de poblaciones de peces que se desarrollan en el mismo espacio en el mismo tiempo" Sin embargo, esta definición está incompleta, porque está basada en la situación de la marea baja. En marea alta, otras muchas especies migran hacia áreas que previamente estaban emergidas, y algunas de ellas pueden interactuar con los miembros de la comunidad residente. (INVEMAR, 2005)

Las comunidades ícticas intermareales presentan una distribución espacial agregada, esto implica que los individuos se agrupan en aglomerados o parches, dejando porciones del espacio relativamente desocupadas. Estos aglomerados están a menudo separados por extensas playas de arena. Esta segregación explica que las comunidades intermareales de peces, y las poblaciones que las constituyen, sean prácticamente unidades independientes y si están conectadas, lo están por difusión de poblaciones a lo largo de la zona rocosa submareal o por estrechos corredores que conectan esos parches intermareales, sin que esta separación implique que las comunidades sean diferentes en términos taxonómicos o ecológicos. (COMPAIRÉ, 2012)

Los peces que utilizan el intermareal como hábitat, lo hacen durante períodos de tiempo diferentes o en diferentes momentos de su ciclo de vida. En un extremo hay especies que viven allí durante toda su vida; por otro lado, las hay que entran sólo endeterminados períodos durante la marea alta. En el intermareal rocoso, algunas veces, las pozas contienen un gran número de juveniles de especies que son predominantemente submareales, lo que sugiere que el intermareal actúa como zona de alevinaje para estas especies, actuando como refugio 
temporal y espacial ante los depredadores, que son más grandes y numerosos en la zona submareal.(Gonzales, 2010)todos hacen la distinción básica entre habitantes permanentes (residentes) y otros que visitan la zona intermareal en algún momento de su ciclo vital de visitantes.

En las costas rocosas, las especies residentes verdaderas son de pequeño tamaño, con formas crípticas que muestran numerosas adaptaciones (morfológicas, fisiológicas y de comportamiento) a la vida intermareal. Muchas son territoriales y suelen vivir entre las algas, en refugios o en microhábitats muy específicos.

La dieta y el comportamiento trófico de muchas de estas especies están poco estudiados pero la mayoría son predadores visuales, probablemente diurnos. Así mientras algunas especies desarrollan todo su ciclo vital en el intermareal, hay otras, las denominadas residentes parciales, que migran a aguas más profundas donde completan su ciclo de vida una vez alcanzado un tamaño o un estado de madurez determinado. Las especies visitantes estacionales son aquellas que utilizan estas zonas para la puesta, por lo que forman parte de estos ensamblajes sólo en el momento de la reproducción. Por último, se encuentran las especies visitantes de marea, que utilizan el intermareal como área de alimentación. La proporción relativa de especies residentes y visitantes puede variar estacionalmente por lo que se debe tener en cuenta la época en la que se han recolectado los ejemplares. (Quijada, 2000)

\section{CONCLUSIONES}

Se encontraron 17 especies siendo las abundante la Lorna seguido de la Chita y luego los borrachos y los Tramboyos la Corvina, con la peculiaridad que las Lornas y las chitas están con sus hueveras una talla pequeña, produciendoce lo que se llama la juvanación que no es más que la defensa de estas especies para poder existir. Las especies están enumeradas por su nombre común o vulgar y queda compromiso poner el nombre científico y aún están en proceso.

\section{REFERENCIAS BIBLIOGRÁFICA}

COMPAIRÉ, J. C. (20 de DICIEMBRE de 2012). https://www.researchgate.net/...intermareal.../558 000b308aeb61eae272766.pdf. Obtenido de https://www.researchgate.net/...intermareal.../558 000b308aeb61eae272766.pdf: https://www.researchgate.net/...intermareal.../558 000b308aeb61eae272766.pdf

GEF-ONUDI-IMARPE-IFOP. (20 de octubre de 2002). humboldt.iwlearn.org/es/informacion.../GEFMOD ULOIIRecursosypesqueriasVol1.pd... Obtenido de humboldt.iwlearn.org/es/informacion.../GEFMOD ULOIIRecursosypesquerias Vol1.pd... : humboldt.iwlearn.org/es/informacion.../GEFMOD ULOIIRecursosypesqueriasVol1.pd...

Gil, E. M. ( 2006). Ictiofauna intermareal de una plataforma rocosa del Golfo de Cádiz:. Cádiz: Servicio de Publicaciones de la Universidad de Cádiz.

Gonzales, C. (4 de setiembre de 2010). www.academia.edu/.../PATRONES DE ABUND ACIA_Y_DIVERSIDAD_DE_LA_I.. Obtēnido de www.āāemia.edu/.../PATRONONES_DE_ABUND ACIA_Y_DIVERSIDAD_D E_ LA_ I . : www.acade-mia.edu/.../PATRONES_DE_ABUND ACIA_Y_DIVERSIDAD_DE_LA_I..

INDECI. (09 de 03 de 2007). google. Obtenido de c i u d a d e s sos t e n i b l e s : http://bvpad.indeci.gob.pe/doc/estudios_CS/Regio n_Lima/huaura/huacho.pdf

INVEMAR, J. B. (2005). INFORME DE LOS ESTADOS DE LOS AMBIENTES MARINOS COSTEROS EN COLOMBIA. Santa Marta: INVEMAR.

Kleine, D. ( 2014). Arrecifes coralinos. Australia: University of Exeter, Devon, UK.

Quijada, P. A. (12 de Diciembre de 2000). www.scielo.cl/pdf/rchnat/v73n4/art16.pdf. Obtenido de www.scielo.cl/pdf/rchnat/v73n4/art16.pdf: www.scielo.cl/pdf/rchnat/v73n4/art16.pdf

Reyna, S. S. (04 de 10 de 2014). google. Obtenido de cybertesis.unmsm.edu.pe/bitstream/cybertesis/38 87/1/Valenzuela_rs.: http://cybertesis.unmsm.edu.pe/bitstream/cyberte sis/3887/1/Valenzuela_rs.pdf 